\title{
Desenvolvimento de larvas de surubim-do-iguaçu (Steindachneridion melanodermatum) submetidas a diferentes dietas
}

\author{
Aldi Feiden ${ }^{1}$, Carmino Hayashi², Wilson Rogério Boscolo ${ }^{3}$ \\ 1 UNIOESTE/Campus de Toledo, Rua da Faculdade 645, J. La Salle, CEP: 85903-000; GEMAq (Grupo de Estudos em Manejo na Aqüicultura). \\ ${ }^{2}$ Curso de Biologia da Universidade Estadual de Maringá - Maringá/PR. \\ ${ }^{3}$ Curso de Engenharia de Pesca - Universidade Estadual do Oeste do Paraná - Campus de Toledo.
}

RESUMO - Para verificar o desenvolvimento de larvas de surubim-do-iguaçu (Steindachneridion melanodermatum) alimentadas com diferentes dietas, realizou-se um experimento em delineamento inteiramente casualizado, com cinco tratamentos e quatro repetições, em aquários de $35 \mathrm{~L}$, cada um com 15 larvas $(8,35 \pm 0,85 \mathrm{~mm}$ e 6,75 $\pm 0,64 \mathrm{mg})$. Os tratamentos consistiram de cinco programas de alimentação: A - Artemia; $\mathrm{R}$ - ração; $\mathrm{Z}$ - zooplâncton; A+R - Artemia + ração; $\mathrm{Z}+\mathrm{R}$ zooplâncton+ração. Avaliou-se o desenvolvimento das larvas em ambiente iluminado durante 29 dias. Para avaliação da dieta e análise do conteúdo estomacal, a cada três dias foram coletadas larvas em cinco aquários adicionais. Nos tratamentos $A+R$ e $\mathrm{Z}+\mathrm{R}$, os pesos médios finais das larvas foram significativamente maiores (659 e $571 \mathrm{mg}$ ) que nos outros tratamentos. A combinação de alimentos possibilitou os melhores resultados e, no tratamento R, a sobrevivência foi de $1,7 \%$, diferindo dos demais. O canibalismo foi superior no R e no Z, que apresentaram taxas de 60 e $25 \%$, respectivamente. O fornecimento de Artemia em associação à ração promoveu melhor desenvolvimento e maior sobrevivênciaàs larvas de Steindachneridion sp.

Palavras-chave: alimentação, alimentos inertes, larvicultura, seletividade alimentar, zooplâncton

\section{Development of Iguaçu surubim (Steindachneridion melanodermatum) larvae fed different diets}

\begin{abstract}
An assay was conducted to evaluate the development of Iguaçu surubim ( Steindachneridion melanodermatum ) larvae fed different diets. The experiment was analyzed as a complete randomized design with five treatments and four replicates, in $35 \mathrm{~L}$-aquaria, each one with 15 larvae $(8.35 \pm 0.85 \mathrm{~mm}$ and $6.75 \pm 0.64 \mathrm{mg})$. The treatments consisted of five feeding programs:Artemia(A); diet (D); zooplankton (Z);Artemia with $\operatorname{diet}(\mathrm{A}+\mathrm{D})$; and zooplankton with diet (Z+D). Larvae development was evaluated in illuminated environment during 29 days. For larvae feeding evaluation, three larvae were collected every three days, in five additional aquaria for stomach content analysis. In the $\mathrm{A}+\mathrm{D}$ and $\mathrm{Z}+\mathrm{D}$ treatments, the larvae average weight ( 659 and $571 \mathrm{mg}$ ) was significantly higher than the other treatments. The combinations of feeds resulted in better results and, in treatment $\mathrm{R}$, it was observed survival rate of 1.7, that differs from the other treatments. Cannibalism rate was higher in $\mathrm{D}$ and $\mathrm{Z}$ treatments, with values of 60 and $25 \%$, respectively. The $\mathrm{A}+\mathrm{D}$ treatment provided the best development and the highest survival rate for Steindachneridion sp larvae.
\end{abstract}

Key Words: feeding selectivity, feeding, inert food, larviculture, zooplankton

\section{Introdução}

Os rios de planalto da bacia do rio Paraná apresentam ictiofauna com muitas espécies endêmicas, possibilitada pelo isolamento geográfico provocado por corredeiras e cachoeiras. O rio Iguaçu, um dos grandes tributários da margem esquerda do rio Paraná (Julio Jr. et al., 1997), caracteriza-se pelo alto endemismo e pela diversidade específica (Sampaio, 1988), observando-se que, em áreas como o médio Iguaçu, $80 \%$ da ictiofauna é formada por espécies endêmicas (Reservatório, 2002). Embora a maioria das espécies seja de pequeno porte, destaca-se o pimelodídeo surubim-do-iguaçu (Steindachneridion melanodermatum), que pode atingir até $70 \mathrm{~cm}$ de comprimento e $15 \mathrm{~kg}$ (Copel, 2001). A espécie com registro recente (Garavello, 1991), é encontrada somente a jusante do reservatório de Segredo (Garavello et al., 1997; Garavello, 2005), sendo rara nas capturas e restrita ao baixo Iguaçu (Severi \& Cordeiro, 1994). Esta região encontra-se muito impactada pelos represamentos e pela intensa ocupação antrópica da região, o que coloca a espécie em risco de extinção.

Nakatani et al. (2001) citaram que existem poucos estudos sobre sua biologia, principalmente na fase inicial de desenvolvimento. Para evitar o desaparecimento global da 
espécie, são necessárias ações no ambiente natural (Agostinho et al., 2002b). Para a produção de juvenis, é importante o conhecimento de seu desenvolvimento, pois a espécie apresenta bons resultados em cultivos experimentais, como demonstrado por Feiden et al. (2001a, b).

A disponibilidade de alimento vivo, com alto valor biológico (Furuya et al., 1999), é essencial, pois o alimento vivo fornece todos os nutrientes essenciais para o desenvolvimento inicial das larvas (Herbert, 1978). As enzimas necessárias à digestão nesta fase muitas vezes ainda não são produzidas, por não haver ainda o diferenciamento total do trato digestório (Dabrowski \& Glogowski, 1977; Verreth et al., 1992).

O zooplâncton é o principal recurso utilizado pelas larvas na natureza (Gerking, 1994), porém, em ambientes controlados, seu cultivo se torna oneroso. Além disso, a coleta de plâncton silvestre é sazonal, podendo modificar quanto à densidade e à composição (Radünz Neto, 1999). O uso de cistos de Artemia para fornecimento de náuplios no início da alimentação exógena é comum, pois proporciona bons resultados (Verischelle et al., 1990; Stickney, 1994; Behr, 1997). Kossowski (1991) recomendou o fornecimento de náuplios de Artemia na primeira alimentação e Sorgenloos et al. (2001) destacaram que Artemia é essencial na larvicultura de espécies marinhas.

A combinação de dietas naturais e artificiais é muito empregada, pois, após a alimentação natural, dietas formuladas tem apresentado resultados excelentes quanto ao desempenho e à sobrevivência (Hogendoorn, 1980; Verreth \& van Tongeren, 1989; Fermin \& Bolívar, 1991; Kerdchue \& Legendre, 1995; Qin et al., 1997; Roselund et al., 1997).

O fornecimento de dietas diferenciadas permite a seleção dos alimentos conforme a preferência, o tamanho ou a fase ontogenética dos peixes, observando-se que, inicialmente, o tamanho da boca é o mecanismo limitante (Zaret, 1980; Lazzaro, 1987). Inicialmente, as larvas selecionam pequenos organismos do plâncton, mas, com o aumento do tamanho da larva, a seletividade diminui (Geiger, 1985; Sipaúba-Tavares, 1993; Zuniga \& Escobar, 1993).

A predação intra-específica, considerada um dos prin cipais obstáculos na larvicultura, por reduzir drasticamente a sobrevivência, está relacionada à densidade de estocagem (Smith \& Ready, 1991; Britz \& Pienaar, 1992; Luz \& Zaniboni Filho, 2002), ao tipo de alimento fornecido (Zaniboni Filho \& Barbosa, 1992; Piovezan, 1994; Laurel et al., 2001; Baras \& Jobling, 2002) e ao crescimento desuniforme das larvas (Hecht \& Appelbaum, 1988; Hecht \& Pienaar, 1993; Kubitza \& Lovshin, 1999; Dou et al.,2000).
Este estudo foi realizado com o objetivo de avaliar o desenvolvimento de larvas de surubim-do-iguaçu (S. melanodermatum) durante a fase inicial alimentadas com diferentes dietas.

\section{Material e Métodos}

Para avaliar o desenvolvimento de larvas de $S$. melanodermatum, foi conduzido um experimento nas instalações do Laboratório de Aqüicultura da Universidade Estadual do Oeste do Paraná, campus de Toledo, no período de 08 de outubro a 06 de novembro de 2002.

Os alimentos avaliados foram: Artemia sp., zooplâncton e ração com $38 \%$ de PB. Para eclosão e obtenção de náuplios deArtemia sp., foram utilizadas incubadoras com capacidade para $2 \mathrm{~L}$ de água, mantidas sob iluminação e aeração constante. $\mathrm{O}$ zooplâncton foi coletado e concentrado diariamente por meio do arraste de rede com malha de $70 \mathrm{~mm}$ em viveiros do Centro de Pesquisas em Aqüicultura Ambiental (CPAA-IAP), em Toledo-PR, e a ração fornecida foi formulada para atender às exigências nutricionais de peixes carnívoros (Tabela 1).

Tabela 1 - Composição química da ração experimental Table 1 - Ingredient and chemical compositions of the experimental diets

\begin{tabular}{|c|c|c|c|}
\hline $\begin{array}{l}\text { Ingrediente (\%) } \\
\text { Ingredient }\end{array}$ & $\%$ & $\begin{array}{l}\text { Nutriente } \\
\text { Nutrient }\end{array}$ & $\%$ \\
\hline $\begin{array}{l}\text { Farinha de peixe } \\
\text { Fish meal }\end{array}$ & 30,23 & $\begin{array}{c}\text { Energia digestível } \\
\quad(\mathrm{kcal} / \mathrm{kg}) \\
\text { Digestible energy }\end{array}$ & 3.600 \\
\hline Farelo de soja & 27,66 & $\mathrm{~PB}$ & 38,00 \\
\hline $\begin{array}{l}\text { Soybean meal } \\
\text { Milho } \\
\text { Corn }\end{array}$ & 17,51 & $\begin{array}{l}C P \\
\mathrm{Ca}\end{array}$ & 3,00 \\
\hline $\begin{array}{l}\text { Farinha de vísceras } \\
\text { Poultry by-products }\end{array}$ & 12,50 & $\begin{array}{l}\mathrm{FB} \\
\mathrm{CF}\end{array}$ & 1,68 \\
\hline $\begin{array}{l}\text { Poultry by-products } \\
\text { Óleo de soja } \\
\text { Soybean oil }\end{array}$ & 8,52 & $\begin{array}{l}\text { P total } \\
\text { Total P }\end{array}$ & 1,56 \\
\hline $\begin{array}{l}\text { Fosfato bicalcico } \\
\text { Dicalcium phosphhate }\end{array}$ & 1,46 & EE & 17,56 \\
\hline $\begin{array}{l}\text { Suplemento }(\min +\mathrm{vit})^{1} \\
\text { Supplement }(\text { min }+ \text { vit })\end{array}$ & 1,00 & $\begin{array}{l}\text { Ácido linoléico } \\
\text { Linoleic acid }\end{array}$ & 5,59 \\
\hline $\begin{array}{l}\text { Calcário calcítico } \\
\text { Limestone }\end{array}$ & 0,61 & $\begin{array}{l}\text { Lisina } \\
\text { Lysine }\end{array}$ & 2,42 \\
\hline $\begin{array}{l}\text { Sal comum } \\
\text { Salt } \\
\text { Antioxidante }(\mathrm{BHT})\end{array}$ & 0,50 & $\begin{array}{c}\text { Metinina+cistina } \\
\text { Met }+ \text { cys }\end{array}$ & 1,55 \\
\hline $\begin{array}{l}\text { Antioxidante (BHT) } \\
\text { Antioxidant BHT } \\
\text { Total }\end{array}$ & 0,02 & & \\
\hline
\end{tabular}

${ }^{1}$ Níveis de garantia por quilograma do produto (Guaranty levels per kilogram of product): vit. A - $500.000 \mathrm{UI}$; vit. $\mathrm{D}_{3}-200.000 \mathrm{UI}$; vit. E $-5.000 \mathrm{mg}$; vit. K3 - $1.000 \mathrm{mg}$; vit. B1 - $1.500 \mathrm{mg}$; vit. B2 - $1.500 \mathrm{mg}$; vit. B6 - $1.500 \mathrm{mg}$; vit. B12 - $4.000 \mathrm{mg}$; ác. fólico(folic acid)- $500 \mathrm{mg}$; pantotenato Ca (calcium pantothenic)- $4.000 \mathrm{mg}$; vit. C - $15.000 \mathrm{mg}$; biotina (biotin) - $50 \mathrm{mg}$; Inositol - 10.000; nicotinamida (nicotinamide) - 7.000; colina (choline) - $40.000 \mathrm{mg}$; Co - $10 \mathrm{mg} ; \mathrm{Cu}$ - 500 mg; Fe - 5.000 mg; I - 50 mg; Mn - 1500 mg; $\mathrm{Se}-10 \mathrm{mg} ; \mathrm{Zn}-5.000 \mathrm{mg}$. 
No experimento, 300 larvas foram distribuídas em 20 aquários com capacidade para $35 \mathrm{~L}$ (unidade experimental), em um delineamento inteiramente casualizado, com cinco tratamentos e quatro repetições. Os tratamentos consistiram de cinco programas de alimentação: A - Artemia sp.; $\mathrm{R}$ - ração; Z - zooplâncton; A+R - Artemia sp. + ração; Z+R - zooplâncton + ração. $\mathrm{O}$ zooplâncton foi produzido em tanques de $200 \mathrm{~m}^{2}$ com laterais de concreto e fundo de terra, adubados semanalmente com adubo orgânico, para manter a transparência entre a 15 e $25 \mathrm{~cm}$ pelo disco de Secchi. Os organismos planctônicos fornecidos foram, na sua maioria, pequenos cladóceras e náuplios de copépodas nos estádios 3 e 5 (62\% de cladóceros, $27 \%$ de naúplios e $11 \%$ de outros grupos).

Em cada aquário, foram mantidas 15 larvas com 48 horas de vida, com comprimento total de $8,35 \pm 0,85 \mathrm{~mm}$ e peso médio de 6,75 $\pm 0,64 \mathrm{mg}$. As larvas, doadas pela Estação Experimental de Estudos Ictiológicos de Segredo, da Copel, foram obtidas por desova induzida. As larvas foram alimentadas três vezes ao dia ( $8 \mathrm{~h}, 13 \mathrm{~h} 30$ e $18 \mathrm{~h}$ ), após a sifonagem dos aquários para retirada de excretas e sobras de alimento. Em cada sifonagem, retirou-se, na primeira semana, $10 \%$ do volume e, nas semanas seguintes, $30 \%$ do volume.

Os parâmetros abióticos da água foram monitorados por meio de aparelhos portáteis digitais. Diariamente, pela manhã e à tarde, foi controlada a temperatura (C) e, semanalmente, foram medidos o $\mathrm{pH}$, o oxigênio dissolvido (mg. $\left.\mathrm{L}^{-1}\right)$ e a condutividade elétrica $\left(\mu \mathrm{S} . \mathrm{cm}^{-1}\right)$. Os fatores bióticos foram monitorados diariamente e, ao final do experimento, todos os peixes foram contados, medidos e pesados individualmente para avaliação do comprimento, do peso e do ganho de peso diário e cálculo da mortalidade, do canibalismo e da sobrevivência.

$\mathrm{Na}$ análise, adotou-se o seguinte modelo estatístico:

$$
\mathrm{Y}_{\mathrm{ij}}=\mu+\mathrm{Ti}+\mathrm{eij}
$$

em que: $\mathrm{Y}_{\mathrm{ij}}=$ observação referente ao aquário $\mathrm{j}$ onde se utilizou o tratamento $\mathrm{i} ; \mu=$ constante geral; $\mathrm{Ti}=$ efeito do tratamento $\mathrm{i}$, em que $\mathrm{i}=\mathrm{A}, \mathrm{R}, \mathrm{Z}, \mathrm{A}+\mathrm{R}$ e $\mathrm{Z}+\mathrm{R}$; e eij = erro aleatório associado à observação $\mathrm{Y}_{\mathrm{ij}}$.

Os dados foram submetidos à análise de variância e, no caso de diferenças estatísticas, aplicou-se o teste Tukey a $1,00 \%$ de probabilidade, por meio do programa computacional Minitab for Windows ${ }^{\circledR}$ versão 10.1. Os valores de mortalidade, canibalismo e sobrevivência foram transformados pela expressão $y=\operatorname{arcsen} \sqrt{\frac{\mathrm{x}}{100}}$, sendo $x \mathrm{o}$ valor destas características expresso em porcentagem.

A cada três dias, foram coletados aleatoriamente de cada aquário três indivíduos, os quais foram fixados em solução de formalina a 4\% para as medidas de comprimento e peso e elaboração das curvas de crescimento para a fase inicial. Amostras do plâncton foram coletadas para análises qualitativa e quantitativa. A identificação do zooplâncton foi realizada com base nos estudos de Rocha \& Tundisi (1976) e Lopretto \& Tell (1995).

Para determinação do espectro alimentar das larvas, procedeu-se à análise do conteúdo estomacal, determinando-se o grau de repleção dos estômagos (GR) e os conteúdos dos tratos digestivos, obtidos por meio de microcirurgia sob microscópio estereoscópico e análise do conteúdo em microscópio óptico, observando-se a ocorrência dos itens presentes e dos ítens dominantes, segundo Hynes (1950) e Hyslop (1980). Os organismos encontrados foram divididos em cinco categorias, conforme a densidade: 0 - não encontrado; 1 - 1 a 20\%; 2 - 21 a 40\%; 3 - 41 a $60 \% ; 4$ - 61 a $80 \% ; 5-81$ a $100 \%$.

\section{Resultados e Discussão}

Os valores médios dos parâmetros bióticos do desenvolvimento inicial de larvas de $S$. melanodermatum alimentadas com diferentes dietas são apresentados na Tabela 2 . Foram observadas diferenças $(\mathrm{P}<0,01)$ entre os diferentes tratamentos para o comprimento final, o peso final, o ganho de peso diário, a sobrevivência, a mortalidade e o canibalismo das larvas de surubim-do-iguaçu (S.melanodermatum).

Os melhores resultados foram obtidos no tratamento $A+R$. Os dados finais comprovaram que a combinação dos alimentos naturais com dietas artificiais permitiram desenvolvimento significativamente maior $(\mathrm{P}<0,01)$. Resultados semelhantes foram encontrados por Szlaminska \& Przybyl (1986), em Cyprinuscarpio, Kerdchuen \& Legendre (1995), em Heterobranchus longifilis, e Soares et al. (2000), em Carassius auratus.

A maior homogeneidade e sobrevivência e o maior peso médio final dos juvenis do tratamento $\mathrm{A}+\mathrm{R}$ em relação aos do Z+R está de acordo com os resultados descritos por Fermin \& Bolívar (1991), que verificaram maior desempenho para Clarias macrocephalus ao compararem o fornecimento de Artemia e Cladóceras (Moina sp.), e Appelbaum \& McGeer (1998), que encontraram alta homogeneidade para larvas de Clarias gariepinus.

Durante o experimento (Figura 1), as larvas dos tratamentos A e A+R até o 15으 dia apresentaram os melhores desempenhos, provavelmente pelo fato de a Artemia ter sido preferida na seleção, comprovado pelo grau de repleção (GR) das larvas (Figura 2). O mesmo foi relatado por Kossowski (1991), que encontrou náuplios de Artemia 
Tabela 2 - Valores médios dos parâmetros bióticos de larvas de Steindachneridion melanodermatumalimentadas com diferentes dietas Table 2 - Average values of the biotic parameters of Steindachneridion melanodermatum fed different diets

\begin{tabular}{|c|c|c|c|c|c|c|}
\hline \multirow{2}{*}{$\begin{array}{l}\text { Parâmetro } \\
\text { Parameter }\end{array}$} & \multicolumn{5}{|c|}{ Tratamento } & \multirow{2}{*}{$\mathrm{CV}$} \\
\hline & A & $\mathrm{R}$ & 7 & $A+R$ & $Z+R$ & \\
\hline Comprimento inicial (mm) & $8,35 \pm 0,85^{\mathrm{a}}$ & $8,35 \pm 0,85^{\mathrm{a}}$ & $8,35 \pm 0,85^{\mathrm{a}}$ & $8,35 \pm 0,85^{\mathrm{a}}$ & $8,35 \pm 0,85^{\mathrm{a}}$ & 0,85 \\
\hline $\begin{array}{l}\text { Initial length }(\mathrm{mm}) \\
\text { Comprimento final }(\mathrm{mm}) \\
\text { Final length }(\mathrm{mm})\end{array}$ & $36,0 \pm 0,9^{\mathrm{a}}$ & $19,2 \pm 0^{\mathrm{c}}$ & $29,3 \pm 1,8^{b}$ & $38,0 \pm 0,2^{\mathrm{a}}$ & $36,2 \pm 2,9^{\mathrm{a}}$ & 1,77 \\
\hline $\begin{array}{l}\text { Peso inicial }(\mathrm{mg}) \\
\text { Initial weight }(\mathrm{mg})\end{array}$ & $6,75 \pm 0,64^{\mathrm{a}}$ & $6,75 \pm 0,64^{\mathrm{a}}$ & $6,75 \pm 0,64^{\mathrm{a}}$ & $6,75 \pm 0,64^{\mathrm{a}}$ & $6,75 \pm 0,64^{\mathrm{a}}$ & 0,64 \\
\hline Peso final (mg) & $446 \pm 37,7^{b}$ & $77 \pm 0^{d}$ & $274 \pm 32,5^{\mathrm{c}}$ & $659 \pm 19,8^{\mathrm{a}}$ & $571 \pm 126,6^{\mathrm{a}}$ & 68,74 \\
\hline $\begin{array}{l}\text { Final weight }(\mathrm{mg}) \\
\text { Ganho de peso diário }(\mathrm{mg} / \mathrm{dia}) \\
\text { Daily weigth gain }(\mathrm{mg} / \text { dia })\end{array}$ & $15,1 \pm 1,3^{\mathrm{ab}}$ & $2,4 \pm 0^{\mathrm{cd}}$ & $9,2 \pm 1,1^{\mathrm{c}}$ & $22,5 \pm 0,7^{\mathrm{a}}$ & $19,4 \pm 4,3^{\mathrm{ab}}$ & 2,36 \\
\hline Sobrevivência $(\%)$ & $75 \pm 6,4^{\mathrm{a}}$ & $1,7 \pm 3,4^{b}$ & $80,0 \pm 12,2^{\mathrm{a}}$ & $80,0 \pm 9,4^{\mathrm{a}}$ & $71,7 \pm 8,4^{\mathrm{a}}$ & 8,47 \\
\hline $\begin{array}{l}\text { Survival (\%) } \\
\text { Mortalidade (\%) }\end{array}$ & $11,7 \pm 11,4^{\mathrm{ab}}$ & $38,3 \pm 20,0^{\mathrm{b}}$ & $8,4 \pm 12,6^{\mathrm{ab}}$ & $5,0 \pm 3,4^{\mathrm{a}}$ & $3,4 \pm 3,9^{\mathrm{a}}$ & 11,94 \\
\hline $\begin{array}{l}\text { Mortality (\%) } \\
\text { Canibalismo }(\%) \\
\text { Cannibalism }(\%)\end{array}$ & $13,3 \pm 14,4^{\mathrm{a}}$ & $60,0 \pm 19,7^{\mathrm{b}}$ & $11,7 \pm 1,4^{\mathrm{a}}$ & $15,0 \pm 10,0^{\mathrm{a}}$ & $25,0 \pm 6,4^{\mathrm{ab}}$ & 13,14 \\
\hline
\end{tabular}

*Valores na mesma linha seguidos de mesma letra não diferem estatisticamente $(P<0,01)$ pelo teste Tukey.

* Values in the same row followed by the same letter do not differ $(P<0.01)$ by Tukey test.

Tratamentos: A - Artemia sp.; R - ração; Z - Zooplâncton; A+R - Artemia + ração; Z+R - Zooplâncton + ração.

Treatments: $A$ - Artemia sp.; $R$ - Diet; $Z$ - zooplankton; $A+R$ - Artemia and diet; $Z+R$ - zooplankton and diet.

$$
\begin{array}{lll}
A- & \hat{Y}=48,204 x-40,693 & R^{2}=0,97 \\
R-\quad \hat{Y} & =3,45 x+2,93 & R^{2}=0,96 \\
Z-\quad \hat{Y}=21,251 x-33,08 & R^{2}=0,96 \\
A+R-\hat{Y}=90,349 x-181 & R^{2}=0,86 \\
Z+R-\hat{Y}=58,901 x-116,81 & R^{2}=091
\end{array}
$$

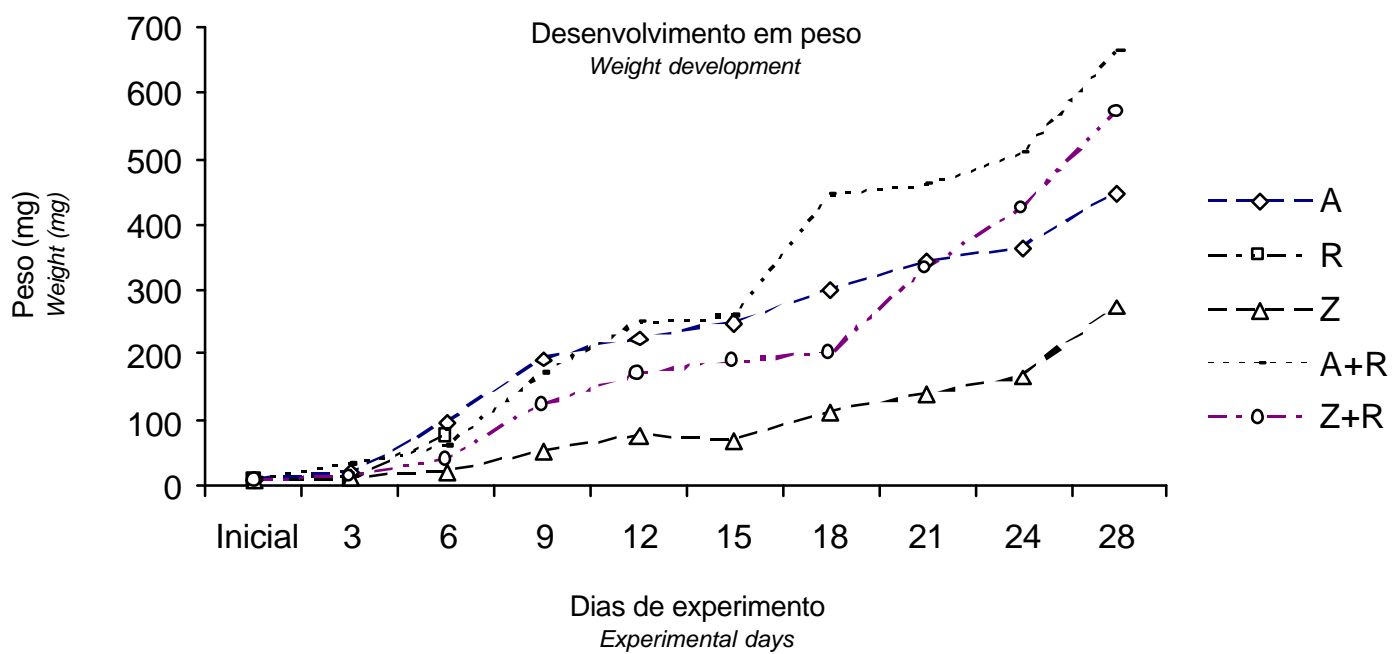

Figura 1 - Curvas de crescimento de larvas de Steindachneridion melanodermatum submetidas a diferentes dietas.

Figure 1 - Curves of growth of experiment 1 with larvae of Steindachneridion melanodermatum fed different diets.

Tratamentos: A - Artemiasp.; R - ração; Z - zooplâncton; A+R-Artemia+ ração; Z+R- zooplâncton + ração.

Treatments: $A$ - Artemia sp.; $R$ - diet; $Z$ - zooplankton; $A+R-$ Artemia $+\operatorname{diet} ; Z+R$ - zooplankton + diet. 


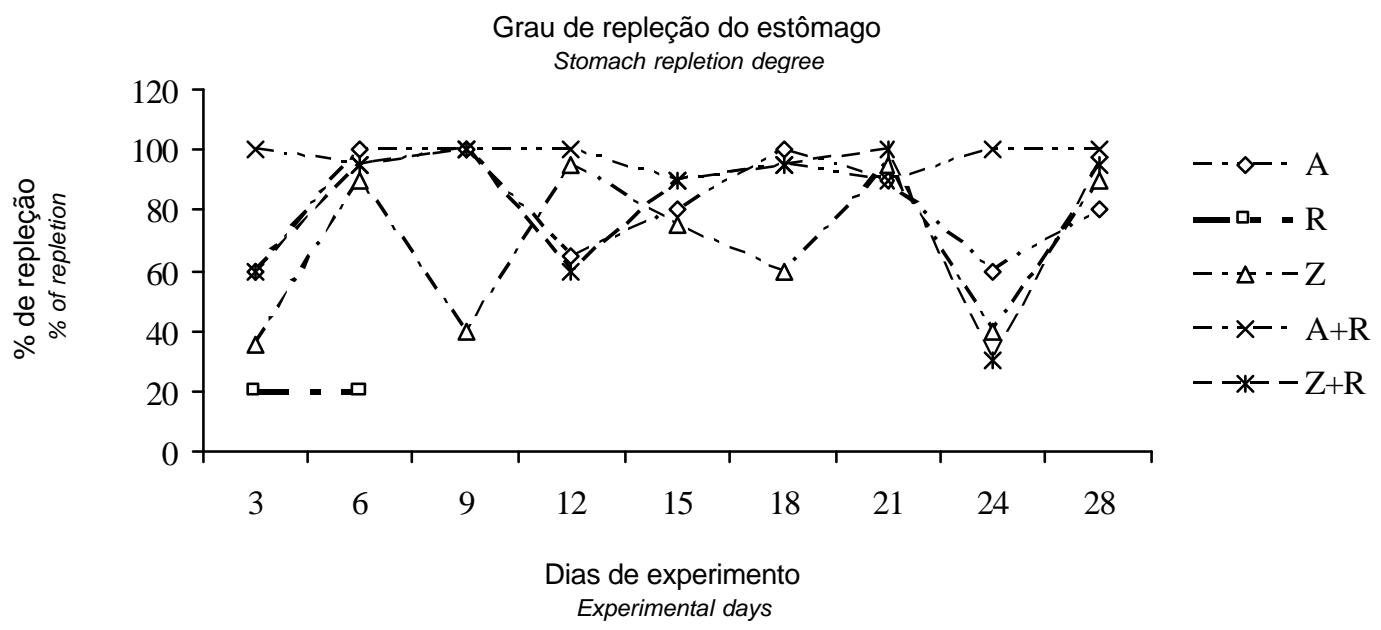

Figura 2 - Efeito do grau de repleção (GR) do estômago sobre a seletividade alimentar e o desenvolvimento de larvas de Steindachneridion melanodermatum.

Tratamentos: A - Artemiasp.; R - ração; Z - zooplâncton; A+R - Artemia + ração; Z+R - zooplâncton + ração.

Figure 2 - Effect of stomach repletion degree (GR) on feeding selectivity and development of Steindachneridion melanodermatum larvae.

Treatments: A - Artemia sp.; $R$ - diet; $Z$ - zooplankton; $A+R$ - Artemia + diet; $Z+R-$ zooplankton + diet.

como primeiro alimento ingerido por Pseudoplatystoma fasciatum e Leiarius marmoratus, e por Naes et al. (1995), em análise dos conteúdos estomacais de Hippoglossus hippoglossus na fase inicial de alimentação exógena.

Ao final do experimento, os peixes do tratamento A tiveram desempenho inferior ao obtido nos tratamentos $\mathrm{A}+\mathrm{R}$ e Z+R, apesar do melhor desenvolvimento até próximo ao $10 \underline{\mathrm{o}}$ dia e terem, durante todo o experimento, os estômagos repletos deArtemia. Esse fato foi comprovado por Baras \& Jobling (2002), que declaram que a passagem da dieta natural para a artificial proporciona maior ingestão de matéria seca (MS) proporcionando desenvolvimento mais rápido. Verreth \& van Tongeren (1989), inclusive, recomendaram, para Clarias gariepinus, fornecer inicialmente alimento vivo por 10 a 14 dias e, posteriormente, passar para dietas artificiais.

O fornecimento exclusivo de alimento artificial no tratamento $\mathrm{R}$ ocasionou menor ganho de peso diário
(24 mg/dia) e comprimento (19,2 mm), como comprovado por Walford \& Lam (1993), que afirmaram que o alimento inerte afeta a sobrevivência e o desenvolvimento pela incapacidade de digestão dos alimentos no início da alimentação exógena, pela composição e pelas características da ração. Além disso, as enzimas digestivas dos organismos-alimento auxiliam a digestão na fase inicial (Dabrowski \& Glogowski, 1977). Baras \& Jobling (2002) relataram que larvas que não se alimentam exaurem suas reservas e morrem por inanição ou ficam suscetíveis à predação, o que justifica o percentual (60\%) de canibalismo (apenas $1,7 \%$ de sobrevivência) e o desenvolvimento inferior em peso e comprimento no tratamento R.

Observou-se predominância de cladóceras nos conteúdos estomacais, indicando a preferência por esses organismos planctônicos, como conseqüência da composição do plâncton, do pequeno tamanho e da reduzida capacidade de locomoção dos cladóceras, o que dificulta o escape

Tabela 3 - Valores médios das características físico-químicos da água Table 3 - Average values of water physical-chemical characteristics

\begin{tabular}{|c|c|c|c|c|c|}
\hline $\begin{array}{l}\text { Parâmetro } \\
\text { Parameter }\end{array}$ & A & $\mathrm{R}$ & $\mathrm{Z}$ & $A+R$ & $\mathrm{Z}+\mathrm{R}$ \\
\hline Temperatura $\left({ }^{\circ} \mathrm{C}\right)$ & $24,7 \pm 0,8$ & $24,9 \pm 0,8$ & $25,0 \pm 0,4$ & $25,2 \pm 0,5$ & $24,7 \pm 0,4$ \\
\hline $\begin{array}{l}\mathrm{pH} \\
\text { Condutividade elétrica }\left(\mu \mathrm{S} . \mathrm{cm}^{-1}\right)\end{array}$ & $\begin{array}{l}7,69 \pm 1,5 \\
140 \pm 3,5\end{array}$ & $\begin{array}{l}7,26 \pm 0,1 \\
117 \pm 1,3\end{array}$ & $\begin{array}{l}7,31 \pm 0,2 \\
114 \pm 1,3\end{array}$ & $\begin{array}{l}7,22 \pm 0,1 \\
147 \pm 5,5\end{array}$ & $\begin{array}{r}7,13 \pm 0,1 \\
116 \pm 1,1\end{array}$ \\
\hline
\end{tabular}

Tratamentos: A - Artemia sp.; R - ração; Z - zooplâncton; A+R-Artemia + ração; Z+R - zooplâncton + ração. Treatments: $A$ - Artemia sp.; $R$ - diet; $Z$ - zooplankton; $A+R$ - Artemia and diet; $Z+R$ - zooplankton and diet. 
(Esteves, 1988). A preferência inicial por cladóceras foi confirmada por Zuniga \& Escobar (1993), em estudo com Cauque maleanum; Rossi (2001), em pesquisa com Sorubim lima; Mackrakis et al. (2005), com Iheringichthys labrosus e Hypophthalmus edentatus em ambientes naturais e em laboratório; Ribeiro et al. (2001), com Leporinus macrocephalus; Sipaúba-Tavares (1993), com Colossoma macropomum; Geiger et al. (1985), com Morone saxatilis; Furuya (2002), com P. corruscans, e por Soares et al. (1997), com Rhinelepis aspera.

De acordo com Cohen \& Lough (1983), a seletividade alimentar pode variar com a composição do plâncton disponível e, quando os cladóceras são mais abundantes, os copépodas são rejeitados.

$\mathrm{Na}$ Figura 2 verifica-se que o GR ao longo do período nos tratamentos $\mathrm{Z}$ e $\mathrm{Z}+\mathrm{R}$ variou de 20 a $100 \%$ de repleção, ao contrário do que ocorreu no A e A+R (60 a $100 \%$ de repleção), o que pode comprovar a maior heterogeneidade dos indivíduos ao final do experimento. Essa variação na taxa de alimentação pode ser explicada pela variabilidade das presas encontradas no ambiente, que, segundo Radünz Neto (1999), apresenta grande variação temporal. Além disso, de acordo com Holmefjord et al. (1993), o zooplâncton silvestre apresenta composição instável, com dominância de espécies não desejáveis. A baixa capacidade de predação na fase inicial de alimentação exógena (nesta fase, os tratamentos nos quais as larvas foram alimentadas com zooplâncton apresentaram grande variação na captura das presas) pode ter influenciado o desenvolvimento.

As larvas que receberam tratamento $\mathrm{Z}+\mathrm{R}$ apresentaram valores médios de peso e comprimento significativamente maiores que aquelas que receberam o tratamento Z. Este resultado confirma os obtidos por Zuniga \& Escobar (1993) em juvenis de Cauque maleanum. Esses autores notaram influência da diminuição da seletividade com o aumento do tamanho da larva e incremento da ingestão de alimento artificial pelas larvas no Z+R (Fermin \& Recometa, 1988; Hayashi et al., 2002).

As altas taxas de sobrevivência observadas, à exceção das larvas submetidas ao tratamento R, permitem afirmar que esta espécie, quando em ambiente com abundância de alimento, apresenta baixa taxa de canibalismo. Pienaar (1990) atestou que o canibalismo pode ser controlado pela alteração da disponibilidade do alimento. Dou et al. (2000) destacaram ainda que o canibalismo é mais freqüente em grupos heterogêneos, confirmando o que ocorreu no tratamento $\mathrm{Z}+\mathrm{R}$.

Os parâmetros abióticos monitorados durante o experimento (Tabela 3) apresentaram valores médios $24,9^{\circ} \mathrm{C}$ para temperatura da água, 7,48 mg.L $\mathrm{L}^{-1}$ para oxigênio dissolvido, 7,33 para $\mathrm{pH}$ e $127 \mathrm{mS} . \mathrm{cm}^{-1}$ para condutividade elétrica. Segundo Sipaúba-Tavares (1994), estes valores estão dentro da faixa de conforto para espécies tropicais.

\section{Conclusões}

O surubim-do-iguaçu S. melanodermatum, quando alimentado com dietas combinadas, apresenta bom desenvolvimento e alta sobrevivência na fase larval. O fornecimento de Artemia tem grande influência na alimentação inicial desta espécie, pois proporciona maior homogeneidade nos juvenis.

\section{Literatura Citada}

RESERVATÓRIO DE SALTO CAXIAS. Bases ecológicas para o manejo. Maringá: UEM/Nupelia/Copel, 2002. 272p. (Relatório técnico final).

AGOSTINHO, A.A.; GOMES, L.C.; SUZUKI, H.I. Reservatório de Salto Caxias: questionamentos feitos pelo PROCAXIAS em relação ao contido no Plano Diretor de Caxias e outros posicionamentos. Parecer Técnico. Maringá: UEM/Nupelia, 2002. 25p. (Mimeo).

APPELBAUM, S.; McGEER, J.C. Effect of a diet and light regime on growth and survival of African catfish (Clarias gariepinus) larvae and early juveniles. Aquaculture Nutrition, v.4, p.157$164,1998$.

BARAS, E.; JOBLING, M. Dynamics of intracohort cannibalism in cultured fish. Aquaculture Research, v.33, p.461-479, 2002.

BEHR, E.R. Efeitos de diferentes dietas sobre a sobrevivência e crescimento de larvas de Pseudoplatystoma corruscans (Agassiz, 1829) (Pisces:Pimelodidae). Maringá: Universidade Estadual de Maringá, 1997. 57p. Dissertação (Mestrado em Ecologia de Ambientes Aquáticos Continentais) - Universidade Estadual de Maringá, 1997.

BRITZ, P.J.; PIENAAR, A.G. Laboratory experiments on the effect of light and cover on the bahaviour and growth of African catfish Clarias gariepinus (Pisces: Clariidae). Journal of Fish Biology, v.227, p.44-62, 1992.

MAKRAKIS, M.C.; NAKATANI, K.; BIALETZKI, A. et al. Ontogenetic shifts in digestive tract morphology and diet of fish larvae of Itaipu Reservoir, Brazil. Environmental Biology of Fishes, v.72, p.99-107, 2005.

CESTAROLLI, M.A.; PORTELLA, M.C.; ROJAS, N.E.T. Efeitos do nível de alimentação e do tipo de alimento na sobrevivência e no desempenho inicial de curimbatá Prochilodus scrofa (Steindachner, 1881). Boletim Instituto de Pesca, v.24, p.119-129, 1997.

COHEN, R.E.; LOUGH, R.G. Prey field of larval herring Clupea harengus on a continental shelf spawning area.Marine Ecology Progress Series, v.10, p.211-222, 1983.

COPEL [2001]. Disponível em <http://www.copel.com/copel/port/ copel.html> Acesso em: 03/10/01.

DABROWSKI, K.; GLOGOWSKI, J. Studies on the role of exogenous proteolytic enzymes in digestion processes in fish. Hydrobiologia, v.54, p.129-134, 1977.

DOU, S.; SEIKAI, T.; TSUMOTO, K. Cannibalism in Japanese flounder juveniles Paralichthys olivaceus, reared under controlled conditions. Aquaculture, v.182, p.149-159, 2000.

ESTEVES, F.A. Fundamentos de limnologia. Rio de Janeiro: Interciência/Finep, 1988. 575p. 
FEIDEN, A.; BOSCOLO, W.R.; REIDEL, A. et al. Proporções corporais do surubim do Iguaçu Steindachneridion $\mathrm{sp}$ (Pisces:Pimelodidae) em três diferentes idades. In: CONGRESSO BRASILEIRO DE ENGENHARIA DE PESCA, 12., 2001, Foz do Iguaçu. Anais... Foz do Iguaçu: AEP/Sul. 2001a. (CD-ROM).

FEIDEN, A.; REIDEL, A.; SIGNOR, A. et al. Desenvolvimento do surubim Steindachneridion $\mathrm{sp}$ em tanques-rede, no reservatório da Hidrelétrica de Salto Caxias. In: CONGRESSO BRASILEIRO DE ECOLOGIA, 5., 2001, Porto Alegre. Resumos... Porto Alegre: Sociedade Brasileira de Ecologia, 2001 b. p. 22 .

FERMIN, A.C.; BOLIVAR, M.E.C. Larval rearing of the philippine freshwater catfish, Clarias macrocephalus (Gunther) fed live zooplankton and artificial diet: a preliminary study. Bamidgeh, v.43, n.3, p.87-94, 1991.

FERMIN, A.C.; RECOMETA, R.D. Larval rearing of bighead carp, Aristichthys nobilis Richardson, using different types of feed and their combinations. Aquaculture Fisheries Management, v.19, n.3, p.283-290, 1988.

FREGADOLLI, C.H. Seleção alimentar de larvas de pacu,Piaractus mesopotamicus Holmberg, 1887, e tambaqui Colossoma macropomum Cuvier, 1818, em laboratório.Boletim Técnico do Cepta, v.6, n.1, p.1-50, 1993.

FURUYA, V.R.B.; HAYASHI, C.; FURUYA, W.M. et al. Influência de plâncton, dieta artificial e sua combinação sobre o crescimento e sobrevivência de larvas de curimbatá (Prochilodus lineatus). Acta Scientiarum, v.21, n.2, p.699703, 1999

FURUYA, V.R.B.; HAYASHI, C.; FURUYA, W.M. et al. Replacement rates of carbon (13C) stable isotope in muscle tissue of pintado, Pseudoplatystoma corruscans (Agassiz, 1829), juvenile. Zootecnia Tropical, v.20, n.4, 2002.

GARAVELLO, J.C. Descrição do gênero Steindachneridion Eigenmann \& Eigenmann, 1919 (Piscis, Ostariophysi, Pimelodidae). In: CONGRESSO BRASILEIRO DE ZOOLOGIA, 1991, Salvador. Resumos... Salvador: Sociedade Brasileira de Zoologia, 1991. p.295.

GARAVELLO, J.C.; PAVANELLI, C.S.; SUZUKI, H.I. Caracterização da ictiofauna do rio Iguaçu. In: AGOSTINHO, A.A.; GOMES, L.C. (Eds.) Reservatório de Segredo: bases ecológicas para o manejo. Maringá: Editora da Universidade Estadual de Maringá, 1997. p.61-84.

GARAVELLO, J.C. Revision of genus Steindachneridion (Siluriformes: Pimelodidae). Neotropical Ichthyology, n.3, v.4, p.607-623, 2005.

GEIGER, J.G.; FITZMAYER, K.; TURNER, C. et al. Feeding habits of larval and fingerling Striped Bass and zooplankton dynamics in fertilized rearing ponds. Progressive FishCulturist, v.47, n.4, 1985.

GERKING, S.D. Larval feeding. In: GERKING, S.D. (Ed.) Feeding ecology of fish. London: Academic Press, 1994. p.139-170.

HAYASHI, C.; SOARES, C.M.; GALDIOLI, E.M. et al. Uso de plâncton silvestre, fermento fresco e levedura desidratada na alimentação de larvas de cascudo chinelo, Loricariichthys platymetopom (ISBRUCHEN F NIJSSEN, 1979)(Osteichhyes: Loricaridae). Acta Scientiarum, v.24, n.2, p.541-546, 2002.

HECHT, T.; APPELBAUM, S. Observations on intraspecific aggression and coeval sibling cannibalism by larval and juvenile Clarias gariepinus (Clariidae: Pisces) under controlled conditions. Journal of Zoology, v.22, n.14, p.21-44, 1988.

HECHT,T.; PIENAAR, A.G. A review of cannibalism and its implication in fish larviculture. Journal World Aquaculture Society, v.24, p.246-261, 1993.

HERBERT, P.D.N. The populations biology of Daphnia (Crustacea: Daphnidae). Biology Review, v.53, p.387-426, 1978.

HOGENDOORN, H. Controlled propagation of the catfish Clarias lazera (Cev). III. Feeding and growth of fry. Aquaculture, v.21, p.233-241, 1980.
HOLMEFJORD, I.; GULBRANDESEN, J.; LIEN, I. et al. An intensive approach to Atlantic halibut fry production.Journal World Aquaculture Society, v.24, n.2, p.275-284, 1993.

HYSLOP, E.J. Stomach content analysis: a review of methods and their application. Journal of Fish Biology, v.17, p.411-429, 1980.

HYNES, H.B.N. The food of freswater slicklbass (Gasterostenes aculeatus and Pygosteus pungitius) with the review of methods used in studies of the food of fishes. Journal of Animal Ecology, v.19, n.1, p.36-56, 1950.

JULIO JR., H.F.; BONECKER, C.C.; AGOSTINHO, A.A. Reservatório de Segredo e sua inserção na bacia do rio Iguaçu In: AGOSTINHO, A.A.; GOMES (Eds.). Reservatório de Segredo: bases ecológicas para o manejo. Maringá:UEM/Copel, 1997. p.1-17.

KERDCHUEN, N.; LEGENDRE, M. Larval rearing of an African catfish Heterobranchus longifilis (Teleostei:Clariidae): a comparison between natural and artificial diet.Aquatic Living Resource, v.17, p.247-253, 1995.

KOSSOWSKI, C. Experiências iniciales sobre la hibridización de Leiarius marmoratus (Gill, 1871) por Pseudoplatystomafasciatum (Linnaeus) (Pises, Siluriformes, Pimelodidae).Acta Cientifica Venezolana, v.42, p.48-50, 1991.

KUBITZA, J.C.; LOVSHIN, J. Formulated diets. Feeding strategies and cannibalism during intensive culture of juvenile carnivorous fishes. Reviews in Fish Science, v.7, p.1-22, 1999.

LAUREL, B.J.; BROWN, J.A.; ANDERSON, R. Behaviour, growth and survival of redfish in relation to prey availability.Journal of Fish Biology, v.59, n.4, p.884-901, 2001.

LAZZARO, X. A review of planktivorous fishes: their evolution, feeding behaviors, selectivities and impacts. Hydrobiologia, v.146, p.97-167, 1987.

LOPES, R.N.M.; SENHORINI, J.A.; SOARES, M.C.F. Crescimento e sobrevivência de larvas de matrinxã Brycon cephalus Günther, 1869 (Pisces:Characidae) sob diferentes dietas alimentares. Boletim Técnico do Cepta, v.7, p.41-48, 1994.

LOPRETTO, E.C.; TELL, G. Ecossistemas de aguas continentales. Metodologías para su estúdio. La Plata: Sur, 1995. 895p.

LUZ, R.K.; REINALTE-TATAGE, D.A.; FERREIRA, A.A. et al. Desenvolvimento embrionário e estágios larvais do mandiamarelo Pimelodus maculatus. Boletin Instituto Pesca, v.27, n.1, p.49-56, 2001.

LUZ, R.K.; ZANIBONI FILHO, E. Larvicultura do mandi-amarelo Pimelodus maculatus Lacépède, 1803 (Siluriformes:Pimelodidae) em diferentes densidades de estocagem nos primeiros dias de vida. Revista Brasileira de Zootecnia, v.31, n.2, p.560$565,2002$.

NAKATANI, K.; AGOSTINHO, A.A.; BAUMGARTNER, G. et al. Ovos e larvas de peixe de água doce: desenvolvimento e manual de identificação. 1.ed. Maringá: Editora da Universidade Estadual de Maringá, 2001. v.1, 389p.

NAESS, T.; GERMAIN-HENRY, M.; NAAS, K.E. First feeding of Atlantic halibut (Hippoglosus hippoglossus) using different combinations of Artemia and zooplankton. Aquaculture, v.130, p.235-250, 1995.

PIENAAR, A.G. A study of coeval sibling cannibalism in larval and juvenile fishes and its control under culture conditions. Grahamstown: Rhodes University, 1990. 162p. Thesis (Master) Rhodes University, 1990.

PEDREIRA, M.M.; SIPAÚBA-TAVARES, L.H. Effect of ligth green and dark Brown colored tanks on survival rates and development of tambaqui larvae Colossoma macropomum (Osteichthyes:Serrassalmidae). Acta Scientiarum, v.23, n.2, p.521-525, 2001

QIN, J.; FAST, A.W.; DEANOA, D. et al. Growth and survival of larval snakehead (Channa striatus) fed different diets. Aquaculture, v.148, p.105-113, 1997.

RADÜNZ NETO, J.Alimento natural versus ração balanceada na larvicultura de peixes. In: REUNIÃO ANUAL DA 
SOCIEDADE BRASILEIRA DE ZOOTECNIA. 36., 1999, Porto Alegre. Anais... Porto Alegre: Sociedade Brasileira de Zootecnia, 1999. p.119-124.

RIBEIRO, R.P.; HAYASHI, C.; MARTINS, E.N. et al. Hábito e seletividade alimentar de pós-larvas de piavuçú Leporinus macrocephalus (Garavello \& Britski, 1988), submetidas a diferentes dietas em cultivos experimentais. Acta Scientiarum, v.23, n.4, p.829-834, 2001.

ROCHA,O.; TUNDISI, T.M. Atlas do zooplâncton. I. Copepoda. São Paulo: Universidade Federal de São Carlos, 1976. v.1, 68p.

ROSELUND, G.; STROSS, J.; TALBOAT, C. Co-feeding marine fish larvae with inert and live diets. Aquaculture, v.155, p.183$191,1997$.

ROSSI, L.M. Ontogenetic diet shifts in a neotropical catfish, Sorubim lima (Scheider) from the river Parana system. Fisheries Management and Ecology, v.8, p.141-152, 2001.

SAMPAIO, F.A.A. Estudos taxonômicos preliminares dos Characiformes (Teleostei, Ostariophysi) da bacia do rio Iguaçu, com comentários sobre o endemismo dessa fauna. São Carlos: Universidade Federal de São Carlos, 1988. 175p. Dissertação (Mestrado em Genética e Evolução) - Universidade Federal de São Carlos, 1988.

SEVERI, W.; CORDEIRO, A.A. Catálogo de peixes do rio Iguaçu. Curitiba: IAP/GTZ, 1994. 118p.

SMITH, C.; READY, P. Cannibalism in teleost fishes. Reviews in Fish and Fisheries, v.1 p.41-64, 1991.

SIPAÚBA-TAVARES, L.H. Análise da seletividade alimentar em larvas de tambaqui Colossoma macropomum e tambacu (híbrido, pacu - Piaractus mesopotamicus e tambaqui Colossoma macropomum sobre organismos zooplanctônicos. Acta Limnologica Brasiliensia, v.6, p.114-132, 1993.

SIPAÚBA-TAVARES, L.H. Limnologia aplicada à aqüicultura. Jaboticabal: Funep, 1994. 70p.

SIPAÚBA-TAVARES, L.H.; ROCHA, O. Sobrevivência de larvas de Piaractus mesopotamicus (Holmberg, 1887) (pacu) e Colossoma macropomum (Cuvier, 1818) (tambaqui) cultivados em laboratório. Biotemas, v.7, n.1/2,p.46-56, 1994.

SOARES, C.M.; HAYASHI, C.; FURUYA, W.M. et al. Alimentação natural de larvas do cascudo preto Rhinelepis áspera Agassiz, 1829 (Osteichthyes-Loricariidae) em tanques de cultivo. Boletim Instituto de Pesca, v.24, n.esp., p.109-117, 1997.
SOARES, C.M.; HAYASHI, C.; GONGALVES, G.S. et al. Plâncton, Artemia sp, dieta artificial e suas combinações no desnvolvimento e sobrevivência do quinguio (Carassius auratus) durante a larvicultura. Acta Scientiarum, v.22, n.2, p.383$388,2000$.

SORGENLOOS, P.; DRERT, P.; CAWDREVA, P. Use of the brine shrimp Artemia spp. In marine fish larviculture.Aquaculture, v.200, p.147-159, 2001.

STICKNEY, R.R. Principles of aquaculture. New York: John Willey \& Sons, 1994. 502p.

SZLAMINSKA, M.; PRZYBYL, A. Feeding carp (Cyprinus carpio) larvae with an artificial dry food, living zooplankton and mixed food. Aquaculture, v.54, p.77-82, 1986.

VERRETH, J.; TORREELE, E.; SPAZIER, E. et al. The development of a functional digestive system in the African catfish Clarias gariepinus (Burchell). Journal World Aquaculture Society, v.23, p.286-298, 1992.

VERRETH, J.; Van TONGEREN, M. Weaning time in Clarias gariepinus (Burchell) larvae. Aquaculture, v.83, p.81-88, 1989.

WALFORD, J.; LAM, T.J. Development of digestive tract and proteolytic enzyme activity in seabass (Lates calcifer) larvae and juveniles. Aquaculture, v.109, p.187-205, 1993.

ZANIBONI-FILHO, E.; BARBOSA, N.D.C. Larvicultura na CEMIG. In: ENCONTRO ANUAL DE AQUICULTURA DE MINAS GERAIS, 10., 1992, Belo Horizonte. Anais... Belo Horizonte: CEMIG, 1992. p.36-42.

ZARET, T.M. Predation and freswater communities. New Haven: Yale University Press, 1980. 187p.

ZUNIGA, L.R.; ESCOBAR, P.A. Selectividad alimentaria de estados juveniles de Cauque mauleanum (Pisces: Atherinidae) em la laguna de Quintero, Valparaiso, Chile. Acta Limnologica Brasiliensia, v.6, p.157-162, 1993. 